\title{
Regulation of Cortical Actin Cytoskeleton and Amoeboid Motility through the mDia2/DIP Complex
}

\author{
K.M. Eisenmann
}

The University of Toledo Health Science Campus, 3000 Arlington Avenue, Toledo, Ohio 43614

Mammalian cells display remarkable capacities for migration, invasion, and morphological plasticity. These attributes make possible numerous biological processes of central interest in the understanding of development, homeostasis and disease, including metastasis. Metastasis is a major therapeutic challenge- few treatments exist that effectively treat metastatic disease, and metastases are responsible for $>90 \%$ of cancer deaths. Metastatic cancer cells are capable of migration and invasion through extracellular matrix (ECM) barriers present in tissues, intravasation into lymphatics or bloodstream, extravasation, and dissemination and growth at a new site and these processes depend upon dynamic modulation of the cytoskeleton. A thorough understanding of the contribution of proteins regulating the actin cytoskeleton during cell migration and invasion is essential for progress in diagnosis and therapy of metastasis.

Cell motility is driven, in part, by cycles of actin polymerization, cellular adhesion to ECM substrates and actomyosin-based contractility. While the bulk of in vitro studies have been performed upon 2D substrates, these studies do not sufficiently address the importance of the 3D tissue environment, ECM remodeling or cellular architecture, and only account for a single mode of motility in which actin polymerization drives protrusion at the leading edge of the cell, propelling forward motion. Epithelial-derived cells can convert to a mesenchymal-like cell via changes leading to dissociation of tight and adherens junctions to promote detachment of single cells from the epithelial sheet and migration into an adjacent tissue compartment[1-4]. This transition is referred to as the epithelial-mesenchymal-transition (EMT), and plays a role in tumorigenesis. Cellular and molecular changes associated with EMT include loss of E-cadherin expression, MMP upregulation, and adoption of a spindle-like, polarized fibroblast-type of morphology promoting cellular motility/invasion into the surrounding stroma. The mesenchymal-type of 3D migration involves MMP-dependent proteolysis of the surrounding matrices. The expansion of the leading and retraction of trailing edge of the cell is mediated by cortically localized actin and myosin; tightly coordinated, localized actin polymerization and actomyosin-based contraction drives mesenchymal migration, with actin assembly preceding contraction to promote extension of actin-rich protrusions.

Analyses of cell migration in 2D and 3D matrices revealed an additional layer of complexity; tumor cells can utilize two distinct modes of motility- mesenchymal and amoeboid- and their interconvertibility suggests a complex plasticity in mechanisms driving tumor cell movement (Table I). Conversion from the mesenchymal to amoeboid cell migration (ACM) is termed the Mesenchymal-Amoeboid Transition (MAT). ACM was described as a specialized mode of breast cancer cell migration and may play an essential role in metastasis. Amoeboid motility is less dependent upon either MMPs or integrins[5]; amoeboid cancer cells typically have weakened integrin-dependent adhesion and show defects in regulating focal adhesion turnover. Differences in adhesion strength to ECM substrates may promote the higher velocities in 3D migration amongst amoeboid cells. Amoeboid cancer cells have a submembranous cortical F-actin cytoskeleton that is attached to the plasma membrane and is maintained by the activities of Rho proteins and their effecters, such as Rho-kinase (ROCK)[6] and the formin mDia2[7]. Disruptions of the Factin/membrane attachment, hyperactivation of Rho or ROCK, or modulation of Rho-GTPase 
effecters such as mDia2 promote contraction of cortical actin and are associated with dynamic membrane blebbing. Bleb extrusion allows amoeboid cells to squeeze through gaps and adapt the cell body to preexisting spaces in the ECM, or exert sufficient force to deform the ECM fibers[8-10].

The canonical mammalian Diaphanous (mDia)-related Formin-2 regulates the cortical actin cytoskeleton through association with Diaphanous-interacting protein (DIP). Upon interacting with $\mathrm{mDia} 2$, DIP inhibits mDia2's ability to nucleate, processively elongate and bundle non-branched Factin. Direct interaction between the Formin homology-2 (FH2) domain and the leucine-rich DIP Cterminus (LRR)) disrupts the cortical actin cytoskeleton, triggering non-apoptotic plasma membrane blebbing on two-dimensional (2D) matrices. Membrane blebbing is a physiological process engaging elements of the contractile machinery and functions in ACM. Here we demonstrate a tightly regulated complex between $\mathrm{mDia} 2$ and DIP that deregulates $\mathrm{mDia} 2$-dependent $\mathrm{F}$-actin dynamics in vitro and in vivo and induces membrane blebbing. Confocal microscopy reveals that both DIP and mDia2 are dynamically localized to blebs in constitutively blebbing cancer cells in both $2 \mathrm{D}$ and $3 \mathrm{D}$, suggesting a critical role in cortical contractility fundamental to the amoeboid phenotype. Conversely, depleting DIP inhibits ACM and promotes elongated, filopodia-rich morphology in breast cancer cells predisposed to this type of 3D migration. We propose that DIP and $\mathrm{mDia} 2$ comprise a precise regulatory mechanism controlling cortical actin assembly and are required for driving cortical contractility and the amoeboid-type of cancer cell invasion. Understanding the molecular basis of amoeboid cell movement will lend novel insight into mechanisms controlling and contributing to cancer cell migration, invasion, and metastasis and may provide a critically needed alternate therapeutic for metastatic disease.

References

1. $\quad$ Thiery, J.P. \& Sleeman, J.P. Nat Rev Mol Cell Biol 7, 131-142 (2006).

2. Guarino, M. The international journal of biochemistry \& cell biology 39, 2153-2160 (2007).

3. Yilmaz, M. \& Christofori, G. Cancer metastasis reviews 28, 15-33 (2009).

4. Klymkowsky, M.W. \& Savagner, P. Am J Pathol 174, 1588-1593 (2009).

5. Wolf, K. et al. The Journal of cell biology 160, 267-277 (2003).

6. $\quad$ Olson, M.F. \& Sahai, E. Clin Exp Metastasis 26, 273-287 (2009).

7. $\quad$ Eisenmann, K.M. et al. Curr Biol 17, 579-591 (2007).

8. Rosel, D. et al. . Mol Cancer Res 6, 1410-1420 (2008).

9. $\quad$ Wyckoff, J.B., et al., Curr Biol 16, 1515-1523 (2006).

10. Gadea, G. et al., Curr Biol 18, 1456-1465 (2008).

11. This work was supported by DOD Breast Cancer Concept Award \#BC053649

Table 1. Comparison of mesenchymal and amoeboid migration.

\begin{tabular}{|c|c|}
\hline Mesenchymal "path-generating" motility & Amoeboid "path-finding" motility \\
\hline $\begin{array}{l}\text { - } \text { spindle-like, polarized morphology } \\
\text { - } \text { F-actin-rich protrusions including } \\
\text { filopodia, lamellae, invadopodia } \\
\text { - } \text { strong, dynamic ECM adhesion } \\
\text { - } \text { MMP-driven ECM proteolysis creates } \\
\text { tube-like tracts } \\
\text { - Rac signaling maintains actin } \\
\text { meshwork at leading edge } \\
\text { - low migration speed }\end{array}$ & 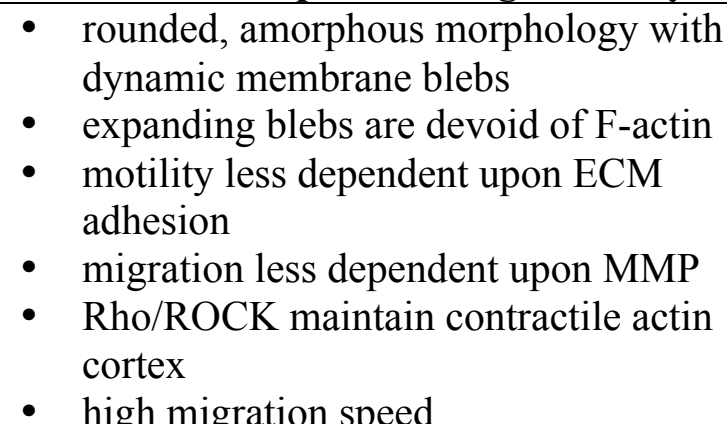 \\
\hline
\end{tabular}

